\title{
Recognition of Isopentenylpyrophosphate and Daudi Tumor Cells By Distinct Subsets of $\mathbf{V} \gamma \mathbf{2} / \mathbf{V} \delta 2 \mathrm{~T}$ Cells
} Andrew Hebbeler* ${ }^{*}$, Cristiana Cairo and C David Pauza

\author{
Address: Institute of Human Virology, University of Maryland Biotechnology Institute, Baltimore, MD 21201
}

* Corresponding author $\ddagger$ Presenting author

from 2005 International Meeting of The Institute of Human Virology

Baltimore, USA, 29 August - 2 September 2005

Published: 8 December 2005

Retrovirology 2005, 2(SuppI I):PI49 doi:I0.I I86/I742-4690-2-SI-PI49

Gammadelta $(\gamma \delta)$ T cells account for $1-10 \%$ of CD3+ lymphocytes in the peripheral blood and mostly express a heterodimeric $\mathrm{T}$ cell receptor (TCR) with $\mathrm{V} \gamma 2^{+}$and $\mathrm{V} \delta 2^{+}$ chains. Although the V $\gamma 2 / \mathrm{V} \delta 2$ subset is defined by the shared expression of common TCR gene segments, these TCRs are highly diverse due to characteristic $\mathrm{N}$ nucleotide insertion and deletion at the complementarity-determining region 3 (CDR3) of both $\gamma$ - and $\delta$-chains. V $\gamma 2 / \mathrm{V} \delta 2 \mathrm{~T}$ cells recognize alkylphosphates that are ubiquitous intermediates in isoprenoid biosynthesis and tumor cells derived from hematopoietic malignancies in a non MHCrestricted, TCR-dependent manner. Previous work from our lab demonstrated that a model alkylphosphate, isopentenylpyrophosphate (IPP), specifically selects $J \gamma 1.2^{+}$ chains and selectively skews the $\mathrm{V} \gamma 2$ repertoire toward longer chain lengths. We assumed that $\mathrm{V} \gamma 2 / \mathrm{V} \delta 2$ recognition of alkylphosphates and tumor cells was common and hypothesized that Daudi B cells, the model tumor target for $V \gamma 2 / V \delta 2 T$ cells, would similarly promote the outgrowth of $\mathrm{V} \gamma 2 / \mathrm{V} \delta 2$ lymphocytes with longer, $\mathrm{J} \gamma 1.2 \mathrm{~V} \gamma 2$ TCRs. Peripheral blood mononuclear cells (PBMC) from 6 donors were stimulated in vitro with interleukin-2 (IL2) alone, IL2 and IPP, or IL2 and irradiated (120 Gy) Daudi tumor cells. The frequency of $\mathrm{V} \gamma 2 / \mathrm{V} \delta 2$ lymphocytes increased from $5.8 \pm 7.8 \%$ on Day 0 to $5.5 \pm 5.9 \%, 34 \pm$ $32 \%, 47 \pm 27 \%$ after 2 weeks in culture with IL2, IL2+IPP or IL2+Daudi, respectively. RNA was extracted before and after stimulation, $\mathrm{V} \gamma 2$ and $\zeta \delta 2$ chains were amplified from reverse transcribed CDNA, and spectratype analysis was performed to assess changes in the distribution of $\mathrm{Vg} 2$ CDR3 lengths. IPP and Daudi similarly skewed the V $\gamma 2$ repertoire toward longer chain lengths, while not affecting the overall distribution of V82 chain lengths. Comparison of $V \gamma 2$ CDR3 sequences from three donors suggest that recognition of IPP and Daudi is mediated by two distinct subsets of $\mathrm{V} \gamma 2 / \mathrm{V} \delta 2 \mathrm{~T}$ cells, thus overturning the prevailing model for gd $\mathrm{T}$ cell recognition of tumors. Collectively, these experiments help clarify the role of V $\gamma 2$ CDR3 specificity in alkylphosphate and tumor recognition and demonstrate that discrete subsets of $\mathrm{V} \gamma 2 / \mathrm{V} \delta 2 \mathrm{~T}$ cells mediate alkylphosphate and tumor responsiveness. 